\title{
Preoperative Chemotherapy
}

National Cancer Institute

\section{Source}

National Cancer Institute. Preoperative Chemotherapy. NCI Thesaurus. Code C68770.

The use of a chemotherapy agent that is administered to a patient prior to the surgical removal of a tumor. 